\title{
Status and Perspectives of the S-DALINAC Polarized-Electron Injector*
}

\author{
M. Herbert ${ }^{\dagger}$, J. Enders, M. Espig, Y. Fritzsche, N. Kurichiyanil, M. Wagner \\ Instit für Kernphysik, Technische Universität Darmstadt, Germany \\ E-mail: mherbert@ikp.tu-darmstadt.de,
}

The S-DALINAC Polarized Injector (SPIN) uses GaAs photocathodes to provide pulsed and/or polarized electron beams for nuclear-structure investigations. Recently, a test facility for PhotoCathode Activation, Test, and Cleaning using atomic-Hydrogen (Photo-CATCH) has been developed. This setup uses an inverted insulator geometry. Tests and optimizations are conducted at Photo-CATCH in order to implement the inverted design at SPIN. We will present the current status of Photo-CATCH, the planned upgrade of SPIN (aimed at an operational voltage of $200 \mathrm{kV}$ ) and future measurements.

XVII International Workshop on Polarized Sources, Targets and Polarimetry

Kaist, South Korea

16-20 October, 2017

${ }^{*}$ Work supported by DFG (GRK 2128 AccelencE), BMBF (05H15RDRB1), and in part by the state of Hesse through the LOEWE center HIC for FAIR

${ }^{\dagger}$ Speaker. 


\section{Introduction}

The Institute for Nuclear Physics (IKP) at Technische Universität Darmstadt operates the superconducting Darmstadt linear accelerator S-DALINAC, a recirculating electron accelerator [1]. It provides beams with energies up to $130 \mathrm{MeV}$ with beam currents of up to $15 \mu \mathrm{A}$ [2] for various nuclear experiments. A DC photocathode electron gun, the S-DALINAC Polarized Injector SPIN [3], has been installed in addition to the existing thermionic electron gun. This source uses photoemission from negative-electron-affinity (NEA) GaAs-based photocathodes to provide both polarized and unpolarized beams with a pre-acceleration of up to $125 \mathrm{keV}$. Two types of cathodes are avaliable: bulk-GaAs cathodes are irradiated with blue laserlight at $405 \mathrm{~nm}$ to generate unpolarized beams, while irradiation of strained-layer superlattice-GaAs cathodes with circularly polarized laserlight at an optimum wavelength of $780 \mathrm{~nm}$ is used for polarized beam generation [4].

Several nuclear-structure studies using polarized electron beams within the energy range of the S-DALINAC are foreseen [5]. Quantum efficiency (QE) and cathode lifetime are important parameters for such experiments. A test stand for Photo-Cathode Activation, Test, and Cleaning using atomic Hydrogen (Photo-CATCH) has been developed and built [6] as a research facility for photocathode performance improvement and photo-gun research and development. An inverted-insulator geometry gun (IIGG) design is tested, producing polarized and unpolarized electron beams with energies up to $60 \mathrm{keV}$. Additionally, a cryogenic photo-gun is currently under development [6].

The photo-gun of SPIN is foreseen to be upgraded to an energy of $200 \mathrm{keV}$ to match a new capture section under development for the S-DALINAC injector [8]. This upgrade will replace the current external insulator design with an IIGG based on the prototype test results at Photo-CATCH. This contribution presents the status and perspectives of SPIN and Photo-CATCH. The first section introduces the Photo-CATCH test stand and gives an overview of current developments. The planned upgrade of SPIN is discussed subsequently. The final section presents future measurements at Photo-CATCH.

\section{Photo-CATCH Test Stand}

In order to conduct independent studies of preparation and handling of photocathodes and research and development of photo-gun prototypes, Photo-CATCH has been designed as a standalone test facility with adjacent beamline. Its present layout, featuring seperate chambers for cathode activation, atomic hydrogen cleaning (AHC), and photo-gun testing, is shown in fig. 1. It also uses the same type of cathode holder as used at SPIN, a so-called puck, in order to provide transferability of designs to SPIN. In order to transfer cathodes used at SPIN, a dedicated ultra-high vacuum (UHV) cathode transport chamber, see fig. 2, has been developed. This vessel also allows cathodes to be stored for long-term vacuum lifetime studies. The facility has a system of four chambers. The Load-Lock Chamber (LLC) is used to load cathodes into the system. A preliminary heat cleaning can be applied here before the sample is transferred to the Atomic Hydrogen Cleaning Chamber (AHCC) for surface cleaning. While a conventional cleaning method of annealing above $600{ }^{\circ} \mathrm{C}$ is used at SPIN, the AHC technique has been demonstrated to provide atomically clean cathode 


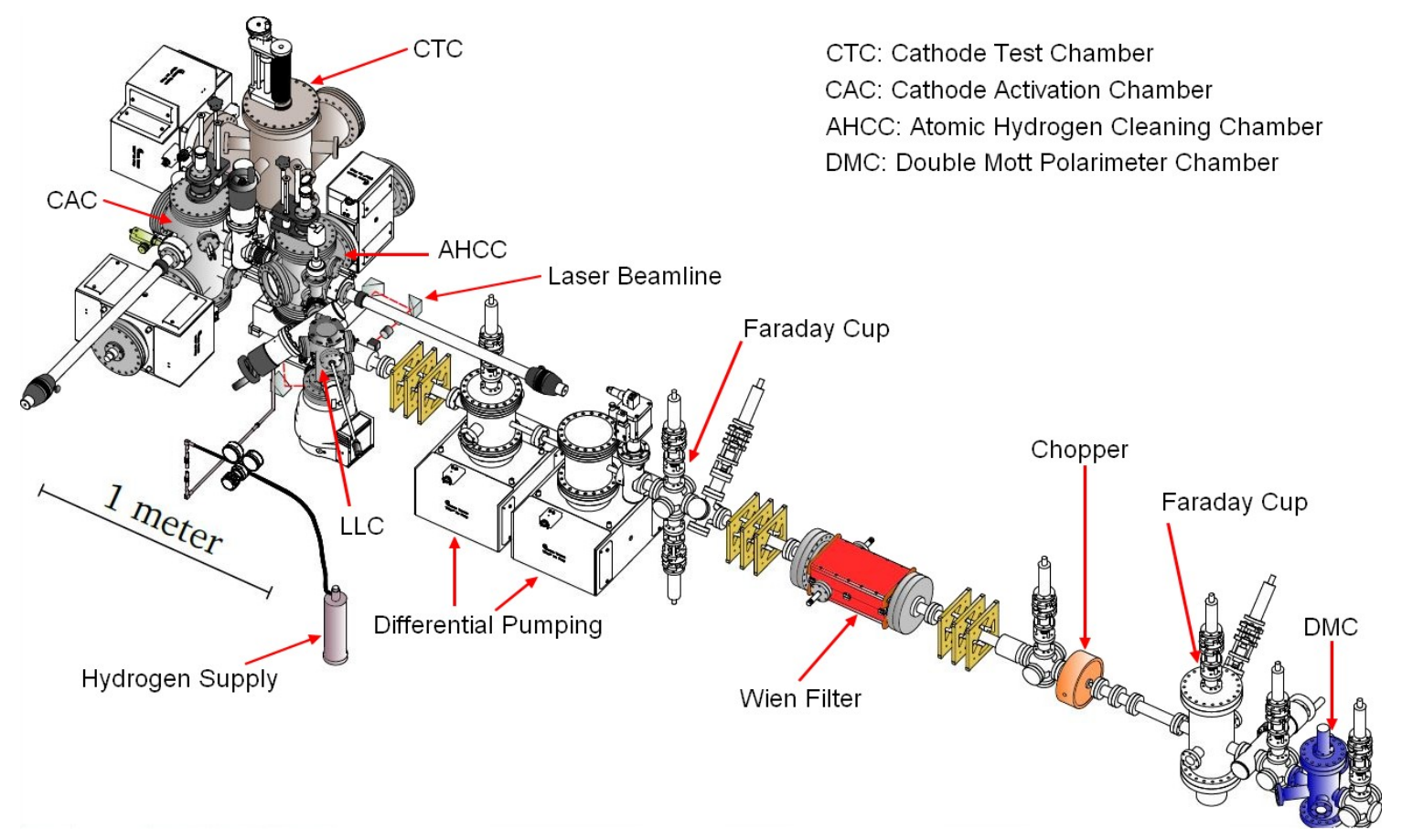

Figure 1: Beamline of the test setup Photo-CATCH. Cathode samples are loaded to LLC and pass through AHCC and CAC before being transferred to CTC for beam extraction.

surfaces at lower temperatures $\left(\sim 400^{\circ} \mathrm{C}\right)$ [6]. The Cathode Activation Chamber (CAC) is able to provide multi-alkali NEA activation, co-depositing lithium ( $\mathrm{Li}$ ) during a traditional caesium (Cs) and oxygen $\left(\mathrm{O}_{2}\right)$ two-stage activation method. This activation scheme is expected to reduce exposure of the active cathode surface to reactive residual gases [9] and an improvement of the activated lifetime by more than a factor of three has been in first experiments [10]. The IIGG is situated in the Cathode Test Chamber (CTC). A schematic of the prototype design is shown in fig 3. It features an electrode with a lift that is used to insert the cathode into the electrode. Mechanical and electrical tests are currently undertaken, aiming at an optimization of the design.

Photo-CATCH is envisaged to be extended by a second photo-electron gun featuring a cryogenic design [7]. Cold surfaces in the vicinity of the cathode are expected to reduce the residual gas pressure, thereby increasing cathode lifetime. This will allow one to conduct investigations on highcurrent operation of GaAs-based spin-polarized electron sources for applications at, e.g., energyrecovery linacs [12] for positron production [13].

\section{Upgrade of SPIN}

Presently, the operation of SPIN with the S-DALINAC requires the optimal operation of a dedicated two-cell capture cavity. In order to increase operability, both this two-cell cavity as well as the regular five cell capture section of the S-DALINAC will be replaced by a reduced-beta design currently under development [8]. The electron-gun of SPIN will be converted to a $200 \mathrm{keV}$ IIGG in order to be compatible to this new capture structure, replacing the present $125 \mathrm{keV}$ external- 

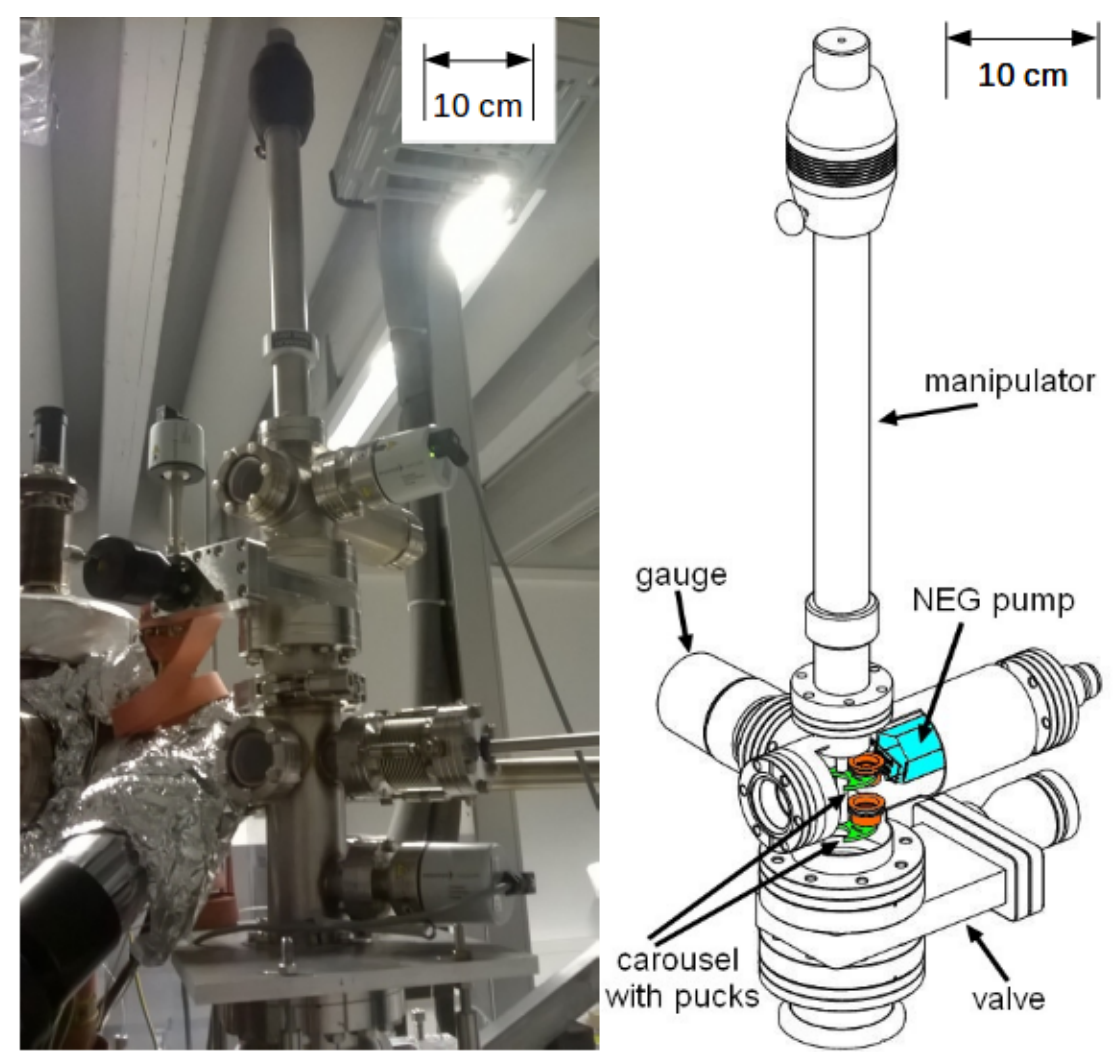

Figure 2: UHV transport vessel, connected to the LLC of Photo-CATCH (left) and schematic drawing (right).

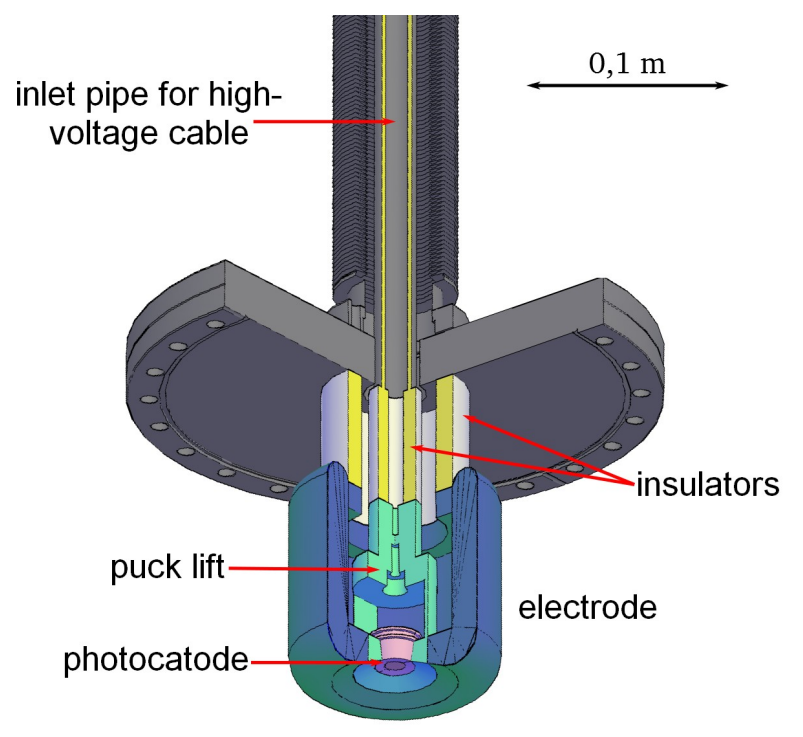

Figure 3: First IIGG prototype design [11]. The puck holding the photocathode is inserted into the lift, which is then retracted into the electrode. High voltage is connected to the lift and transferred to the electrode. 


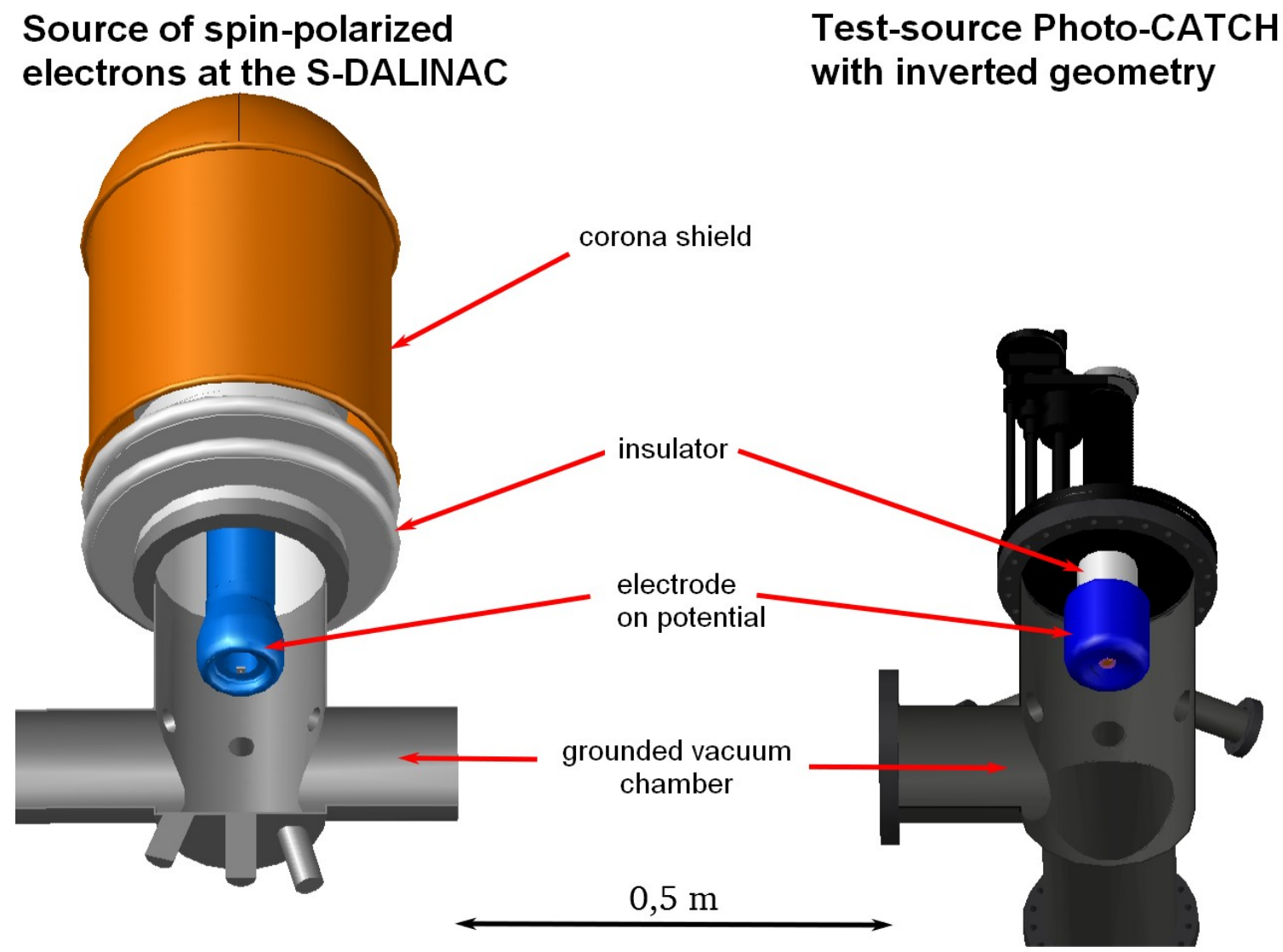

Figure 4: Comparison between gun designs used at SPIN (left) and Photo-CATCH (right [11]).

insulator geometry. The differences between these two designs are shown in fig. 4. Design studies for and operation of the IIGG at Photo-CATCH will be crucial for the planned design at SPIN. The SPIN low-energy beam transfer needs to be adapted accordingly. Recent tests and CST Studio Suite ${ }^{\circledR}$ simulations have shown that the $\alpha$-magnet deflecting the beam into the horizontal beamline can be used at $200 \mathrm{keV}$. The Wien-filter that rotates the electron-spin will be adapted for operation at $200 \mathrm{keV}$. An overhaul of other beamline components, such as steerer and quadrupole magnets, is foreseen as well. The low-energy Mott Polarimeter will be usable at $200 \mathrm{keV}$, as well, albeit with slightly reduced sensitivity.

\section{Proposed Experiments at Photo-CATCH}

The Photo-CATCH test stand provides means to conduct a multitude of studies concerning photo-electron gun design, GaAs photoemission properties, and beam quality. Further cathode lifetime studies for multi-alkali NEA activation are proposed, as well as QE, polarization, beam emittance and bunch structure measurements of such cathodes in the course of the first IIGG and beamline comissioning of Photo-CATCH. Further IIGG design studies testing different electrode materials are also in preparation. Another possible focus for systematic studies is the electron bunch emission process as described by Spicer [14]. Preliminary experiments at SPIN [4] along the lines of Ref. [15] have shown that this description may be insufficient at low QE. 


\section{Conclusion and Outlook}

The photo-electron gun at SPIN is foreseen to be upgraded to a $200 \mathrm{keV}$ IIGG in order to match the new capture cavity structure of the S-DALINAC injector. A dedicated test facility PhotoCATCH has been constructed to provide an environment for research and development of photoelectron guns as well as studies of GaAs-based photocathodes. A first IIGG prototype has been developed and tested, and a second prototype is currently optimized and prepared for tests at PhotoCATCH. Operational experience and results obtained from studies concerning gun design, cathode preparation and handling, photoemission properties and beam parameters will be crucial for future upgrades and operation of SPIN.

\section{References}

[1] A. Richter, "Operational Experience at the S-DALINAC", Proc. of the $5^{\text {th }}$ EPAC, 1996, p. 110.

[2] M. Arnold, T. Kürzeder, N. Pietralla, and F. Hug, in Proc. IPAC'16, Busan, Korea, May 2016, pp. 1717-1719.

[3] Y. Poltaratska et al., J. of Phys.: Conf. Series 298, 012002 (2011).

[4] M. Espig, J. Enders, Y. Fritzsche, and M. Wagner, Proceedings of Science PSTP 2013, 059 (2013).

[5] J. Enders, AIP Conf. Proc. 1563, 223 (2013).

[6] N. Kurichiyanil et al., in Proc. INPAC'15, Mumbai, India, Dec. 2015, paper ID 47.

[7] T. Eggert et al., presented at Proc. PSTP'17, Daejeon, Korea, October 2017, this conference.

[8] D. Bazyl, H. De Gersem, W.F.O. Müller, in Proc. IPAC'17, Copenhagen, Denmark, May 2017, 993.

[9] G.A. Mulhollan et al., J. Vac. Sci. \& Tech. A. 26, 1195 (2008).

[10] N. Kurichiyanil, "Design and construction of a test stand for photocathode research and experiments", Dissertation, Technische Universität Darmstadt, Darmstadt, Germany, 2016.

[11] M. Espig, "Entwicklung, Aufbau und Charakterisierung einer variabel repetierenden, spinpolarisierten Elektronenkanone mit invertierter Isolatorgeometrie", Dissertation, Technische Universität Darmstadt, Darmstadt, Germany, 2016.

[12] T. Rao et al., Nucl. Instrum. Methods in Phys. Research A 557, 124 (2006).

[13] D. Abbott et al., Phys. Rev. Lett. 116, 214801 (2016).

[14] W.E. Spicer, Phys. Rev. 112, 114 (1958).

[15] P. Hartmann et al., J. Appl. Phys. 86, 2245 (1999). 